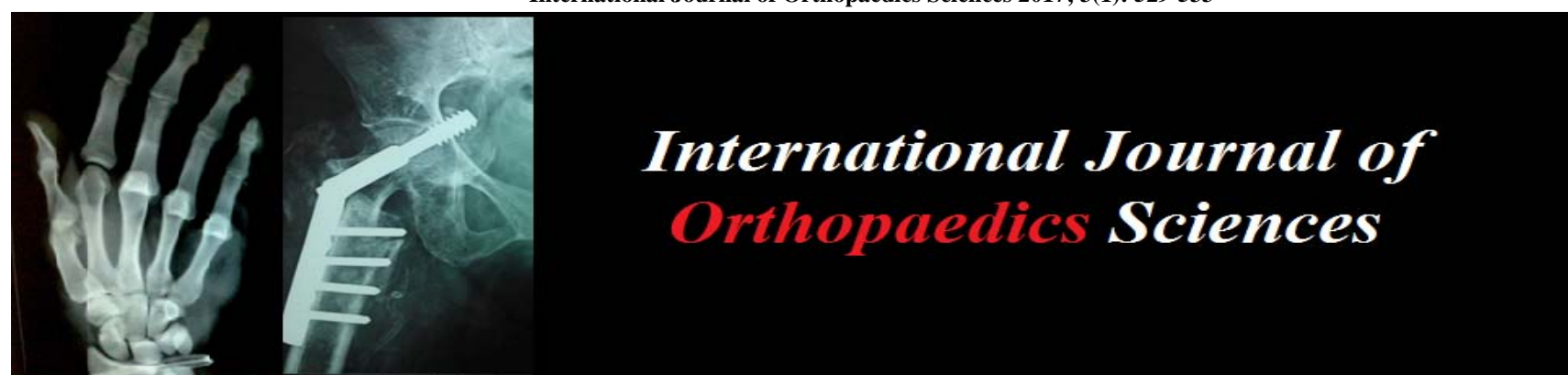

ISSN: $2395-1958$

IJOS 2017; 3(1): 529-533

(C) 2017 IJOS

www.orthopaper.com

Received: 18-11-2016

Accepted: 19-12-2016

Nikhil J

Junior Resident, GMC- Amritsar, India

Partap Singh Verka

Assoc. Prof., GMC- Amritsar,

India

Jaspal Singh

Assoc. Prof., GMC-Amritsar,

India

\section{Role of locking dynamic hip screw in management of inter-trochanteric fractures of femur in elderly patients}

\author{
Nikhil J, Partap Singh Verka and Jaspal Singh
}

DOI: $\underline{\text { http://dx.doi.org/10.22271/ortho.2017.v3.i1h.78 }}$

\section{Abstract}

Introduction: Dynamic Hip screw is the implant of choice for most inter-trochanteric fractures of the femur. But, its most common mode of failure is for the lag screw to cut out of the femoral head, the second is for the plate to be forced off the femur with the screws being pulled out of the osteoporotic bone. With the introduction of Locking plate osteosynthesis, in which fixed angle devices are used, which are not dependent on the holding power of screws alone, the bone-implant construct so formed is mechanically stable and fixation failure due to screw loosening in osteoporotic bone can be reduced. This study aims to evaluate DHS with a locking side-plate for fixation of inter-trochanteric fractures in elderly.

Methods: Aim was to study the outcome of Locking Dynamic Hip Screw fixation in 25 patients admitted in GNDH with inter-trochanteric femur fractures in elderly age group. The clinical and radiological outcomes were evaluated with regular follow-up and the functional grading was done according to Harris Hip Score.

Results: In our study, the mean time to radiological union was 13.96 weeks. There was one patient with deep infection, but no other significant complication in our study. None of the patients in our study had lag screw cut-out, varus collapse or side plate pull out or any other implant specific complications. The average modified harris hip score was calculated to be $82.12 \%$. Functionally, $16 \%$ had excellent, $48 \%$ as good, $32 \%$ as fair and $4 \%$ had poor results.

Discussion: The present study indicates that DHS with a locking side plate is a terrific treatment option for inter-trochanteric fractures in elderly, with no major complications for fracture union. To accomplish the goal of early mobilisation and reduced failures we need an implant which has an inherently stable construct independent of the bone quality and does minimal damage to the vascular supply of the bone and has a low complication rate. Locking DHS helps us achieve that goal.

Keywords: DHS, locking, intertrochanteric, femur, elderly, hip, osteoporosis

\section{Introduction}

With advancement in medical sciences the average life span of humans has risen. As the chunk of elderly patients increase, so does the falls associated with them, which leads to fractures amongst which the most common are the fractures of the proximal part of femur, over half of which are inter-trochanteric fractures. By 2040, the incidence is estimated to be doubled ${ }^{[1]}$. These fractures are also more common in women than in men probably as a result of several factors like wider pelvis in women leading to tendency to coxa vara, women being less active develop osteoporosis which is further enhanced by post-menopausal changes.

All these factors result in an enormous cost on the health system ${ }^{[2,3]}$.

Majority of the trochanteric fractures occur due to direct trauma and major chunk of it due to a simple fall ${ }^{[4]}$. These fractures occurring in elderly patients carry the risk of prolonged immobility and recumbency ${ }^{[5]}$. Therefore the treatment should be so planned as to encourage union without deformity and at the same time allow early mobilisation.

Intertrochanteric fractures associated with communition, osteoporosis and instability often preclude the early resumption of full weight bearing. What must be remembered is that osteoporotic bone has distinct morphologic characteristics that influence its biomechanical properties and therefore the choices and techniques for internal fixation should be well planned

out.

\footnotetext{
Correspondence

Nikhil J

Nikhil J, Junior Resident, GMC-

Amritsar, India
} 
The aim of treatment for intertrochanteric fractures is to make patients ambulatory and return to functional level before trauma in a shortest period of time. The dynamic hip screw (DHS) is the standard implant used for the fixation of intertrochanteric hip fractures ${ }^{[6,7]}$. The most common mode of failure is for the lag screw to cut out of the femoral head, the second is for the plate to be forced off the femur with the screws being pulled out of the osteoporotic bone ${ }^{[8,9]}$. The post-operative cut out ranges from $1 \%$ to $6 \%{ }^{[8]}$.

Most of the clinical and biomechanical studies have studied the effects of optimal placement of lag screw and its angle of insertion, but the side plate fixation has received very little attention $[10,11]$. The side plate in dynamic hip screw is an important factor for anatomical and functional outcome in osteoporotic fractures.

To overcome these problems a locking plate and screw system has been developed. The locking compression plate is the combination of two completely different anchorage technologies in one implant.

Plates secured to bone with locking screws have been used with great clinical success in a number of locations around the body and have been found to produce a stronger construct than standard screws ${ }^{[12]}$. This prevents the implant working loose by screws toggling in the hole in the plate as can occur with non-locking screw.

\section{Materials and Methods}

A prospective interventional study was conducted on 25 patients with inter-trochanteric fractures above the age of 50 years admitted in our hospital. This was done to focus our study on osteoporotic bones in the elderly. Only closed fractures without any neurovascular compromise or intraarticular extension were considered. All patients after admission were thoroughly assessed for any other injury and a comprehensive musculo-skeletal examination along with detailed history taken. Primary stabilization was done.

After primary stabilization of the patient, plain x-ray was done for an AP- view of the pelvis including both the hip joints and also of femur including the knee joint. Skin traction was then applied to immobilise the limb and the necessary investigations done for anesthetic fitness for surgery. The $\mathrm{x}$ ray films were studied and classified according to Boyd and Griffin classification. Since our study involves the elderly, all patients were posted for surgery only after anesthetic clearance and investigations like echocardiography as and when required.

All the patients were operated with DHS with a locking sideplate. Patients were all given a third generation cephalosporin pre-operatively after test dose. Under strict aseptic precautions, under spinal anaesthesia, patient was put on spica table, while the contralateral uninjured leg was placed on a leg holder. Closed reduction was achieved by first giving traction in order to distract the fragments and regain length. This was checked under C-Arm control both AP and laterally. The second step is internal rotation of the leg. Again it was checked under C-Arm in 2 planes as the reduction determines the degree of internal rotation.

A straight approximately $10-12 \mathrm{~cm}$ lateral longitudinal skin incision was made starting at the base of greater trochanter and carrying it distally. Subcutaneous tissue and vastus lateralis were cut along the line of its fibres. A pointed reduction clamp was used to maintain the reduction. In cases of unsatisfactory closed reduction, bone hooks or bone spikes were used to lever the fracture into reduction. A guide wire was then inserted with the help of 135 degree angle guide with the aim to keep it in centre-centre position in c-arm. Importance was given to keep the TAD (Tip-Apex Distance) less than $25 \mathrm{~mm}$ minus the magnification error. The required screw length was then measured and an exclusive DHS triple reamer was employed for the lag screw. Lag screw was the inserted crossing the fracture site and keeping in mind the required TAD. Appropriate 4-6 holed side plate was selected and it was pushed in over the screw and seated home with the impactor. The locking plate was then fixed to the femoral shaft with an appropriate number and size of $4.5 \mathrm{~mm}$ locking cortical screws. Complete hemostasis was achieved and negative suction drain is put and wound closed in layers. Aseptic dressing was done.

Post-operatively, patient's vitals were monitored. Foot end elevation given overnight and drain monitored. Appropriate antibiotics were given. Analgesics were given as per patient compliance. Blood transfused depending upon the preoperative general condition and intraoperative blood loss. Quadriceps strengthening and knee movements were started from the second post-operative day. Mobilization with non-weight bearing started usually after first aseptic dressing change which usually was on the $5^{\text {th }}$ day and continued up to 6-8 weeks depending on the fracture pattern and then partial weight bearing initiated after confirmation of beginning of healing process till fracture union. Full weight bearing was allowed after fracture union. Full stitch out was usually done on $12^{\text {th }}$ post-operative day and patients were called for follow up at 1,2, 3 and 6 months until fracture union. On every visit, ap and lateral view xrays were taken of the fracture site and were examined for union, lag screw position, fracture collapse and any other potential complication. Patients were advised appropriately on weight bearing and other physiotherapy. Functional score was done using modified Harris Hip Score and the results were classified into excellent (90-100), good (80-89), fair (70-79) and poor $(<70)$.

\section{Results}

In our study 25 patients were considered, all above the age of 50 years. Among these the youngest was 52 years old and the oldest 84 years old, with an average of 65.76 years. This was mainly done for the reason to see the effects of locking plate in osteoporotic bones. Females had a higher incidence with 14 $(56 \%)$ of them being females compared to males (44\%).

In our study majority of the patients have suffered injury through fall (19 patients) i.e. $76 \%$ of total. In fact, even assault has resulted in $2(8 \%)$ of them suffering fractures. Type of fracture depends on the mode of injury and also on patient's age, as elderly patients have osteoporotic bones. $36 \%$ of the patients had Boyd and Griffin type 2 fracture, making it the commonest type of fracture in our study. Besides this $8(32 \%)$ patients had type 1 fracture, 3(12\%) had type 3 pattern and $5(20 \%)$ of the patients had type 4 fracture.

Patients were taken for surgery as soon as they were hemodynamically stable and their general condition was good. $8(32 \%)$ patients were operated within 3 days of injury, $14(56 \%)$ patients within 5 days and $3(12 \%)$ patients were operated more than a week later of injury.

Successful fracture union was defined as complete bridging callus in 3 cortices together with painless full weight bearing. Of 25 patients, $10(40 \%)$ patients exhibited radiological union before 12 weeks, $12(48 \%)$ patients exhibited union between 13-16 weeks, 3(12\%) patients showed union between 17-24 weeks. The average radiological union time was 13.96 weeks. No patient reported implant failure or nonunion. 
Our patients were assessed functionally with modified Harris hip Score after fracture union. The average Modified Harris Hip Score in the study was 82.12 with the range of 66.5-92.5. The complications came across were not very severe in nature. $3(12 \%)$ of the patients had superficial wound infections, which responded to antibiotics alone. One (4\%) of them had deep infection for whom prolonged course of IV antibiotics with pus cultures and protected weight bearing was observed. $2(8 \%)$ of them had decubitus ulcers developing which were managed with aseptic dressings along with air beds and increased mobilization. Only one $(4 \%)$ of them had medialisation of shaft for which delayed weight bearing was followed which eventually showed union by 22 weeks. There was more importantly no implant related complication like lag screw cut-out or side plate pull out.

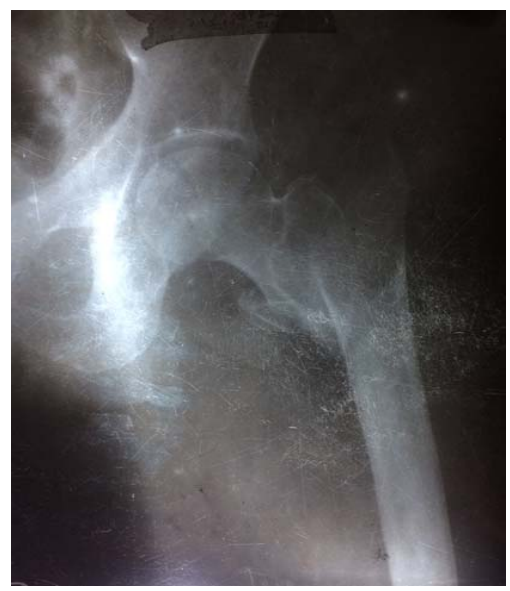

Pre-Operative X-ray

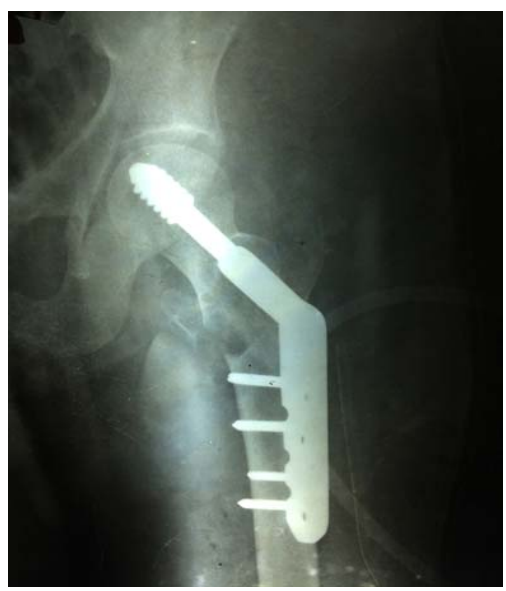

Immediate Post-operative X-ray
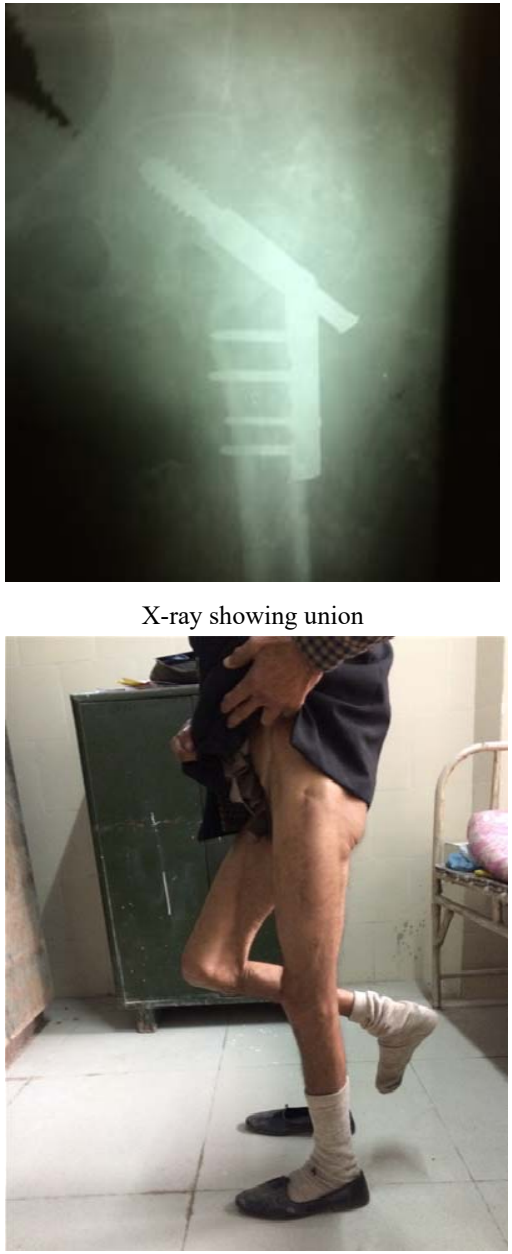

On full weight bearing

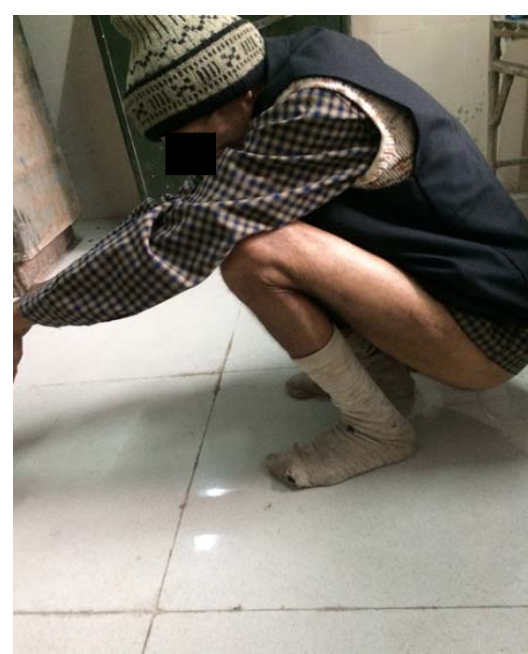

On squatting 


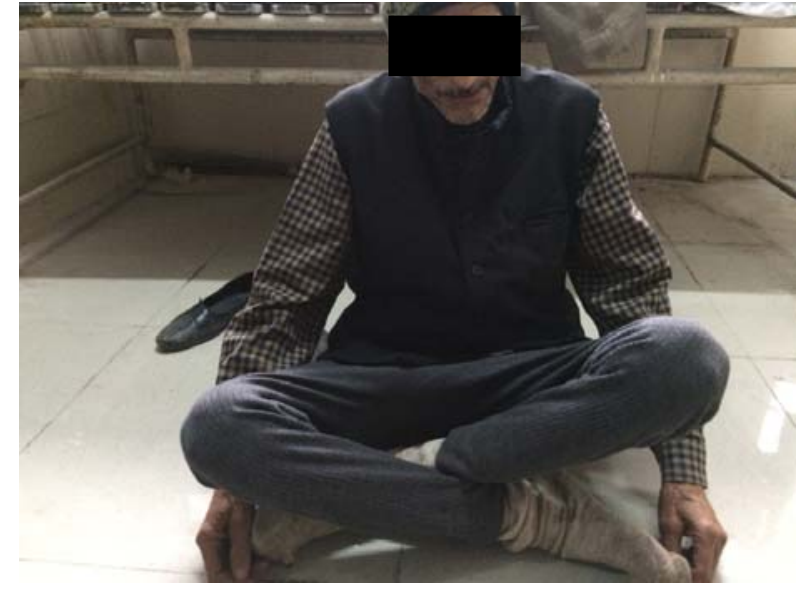

On sitting cross-legged

Case showing union with Locking DHS

\section{Discussion}

In the present study, 25 patients with intertrochanteric fractures of femur above the age group of 50 years were considered. Our sample was such taken that we could do our study on osteoporotic bones. The best treatment for intertrochanteric fractures is debatable, especially for unstable types. The aim of our study was to achieve union at the fracture site and lower the usual complications associated with its fixation. The objective of our treatment is early mobilization and weight bearing so that they can regain their pre-injury functional status. This can be achieved only if the failure rates reduce in unstable fractures and osteoporotic bones of which the elderly are already prone to.

To accomplish such a goal we need an implant which has an inherently stable construct independent of the bone quality and does minimal damage to the vascular supply of the bone and has a low complication rate. Multiple studies have concentrated upon the position of lag screw but the side-plate has been given very little importance. Locking DHS might help us realize that goal.

From a mechanical perspective, decreased bone density and decreased cortical thickness diminish the holding power of screws and lead to fatigue failure of the bone, resorption of the damaged bone, and ultimately to loosening of the implant. There is substantiating evidence of implant failure like lifting off of side-plate, pulling out of screws, screw toggling, screw breakage and cut-out failure of lag screw predominantly occur in osteoporotic bones with less stable fracture configurations ${ }^{[13]}$. This failure precludes early weight bearing and its related complications.

With the introduction of Locking plate osteosynthesis, in which fixed angle devices are used, which are not dependent on the holding power of screws alone, the bone-implant construct so formed is mechanically stable and fixation failure due to screw loosening in osteoporotic bone can be reduced ${ }^{[14-}$ 16]

A dynamic hip screw with fixed angle locking screws would reduce the risk of DHS failure. Locking screw DHS would be particularly useful in patients with poor quality, osteoporotic bone, and in patients with less stable fracture configurations.

The holding power of screws in cortical bone is correlated in a linear fashion to the bone mass which reduces in osteoporotic bones ${ }^{[17]}$.

It has already been proven that biomechanically locking DHS is a more suitable implant than conventional DHS with a significantly higher cut-out resistance, especially in elderly patients with osteoporotic bones ${ }^{[17]}$. Locking plates have biological advantages over the standard plates ${ }^{[18]}$. In osteoporotic bone, normal screws in DHS blade provide less anchorage compared to locking screws. A standard plate grips the bone by the friction created by the compression of the plate against the bone by the screws. This leads to impaired blood supply resulting in decreased cortical thickness and cancellous transformation of the bone. Locking plates do not compress the bone or periosteum, so this weakening should not occur. The risk of peri-implant fractures should therefore be reduced. It has also been suggested that by reducing avascularity within the bone, the risk of infection may be reduced [19]. In a good bone, self-drilling, self-tapping cortical screws would reduce the length of the operation making it safer for the patient. Alternatively, shorter locking plates could be used, providing equivalent strength and requiring less dissection.

In our study, there has been no case of screw cutout or varus collapse or side plate pull out. These are the specific complications seen in a case of intertrochanteric fracture when usually fixed with a conventional DHS with a non-locking side plate, especially more prone in the osteoporotic bones of the elderly. Our results are superior to the non-locking DHS studies where the cutout rates for bridle et al ${ }^{[22]}$. and Baumgartner et al ${ }^{[23]}$. at 3\%. However, locking side plates have a significantly higher pull out resistance as the locking plates do not depend on the screw-bone interface for stability, rather their stability is at the angular stable screw-plate interface and thus acts as a stable mono-construct as a locked internal fixator.

Also, we are re-emphasizing that TAD and neck shaft angle restoration are equally important. These are essential no matter what kind of implant is used. Optimum placement of lag screw: checked in both planes and keeping the TAD $<25 \mathrm{~mm}$ is crucial to prevent any lag-screw migration and attaining a controlled collapse.

Moreover, factors like age of the patient, quality of the bone, nutritional status, compliance to non-weight bearing and mobilization advice particularly in less stable configurations are also important for ultimate fracture outcome. However, locking side plate has definitely minimized the implant related complications like lag screw cut-out, side plate pull out, screw breakage or any bio-mechanical failure. In fact the implant related complications are nil. Additionally, in our study the average union time was 13.96 weeks which is earlier than the conventional DHS studies like Ecker et al ${ }^{[20]}$ (15 weeks), Rao et $a^{[21]}$ (18 weeks). Thus, with earlier union rates, patients can be mobilized early and reduce the recumbency related complications, particularly in old age.

In conclusion, the present study has shown us that locking DHS is a superior option in treating inter-trochanteric fractures in the elderly and the less stable configurations. It gives us healthy bone healing and reduced complication rates, which is essential for a successful outcome and functional outlook for the elderly suffering from such fractures.

\section{References}

1. Kulkarni GS, Limaye R, Kulkarni M, Kulkarni S. Intertrochanteric fractures. Indian J Orthop. 2006; 40:1623.

2. Alffram PA. An epidemiologic study of cervical and trochanteric fractures of the femur in an urban population. Analysis of 1,664 cases with special reference to etiologic factors. Acta orthop scand suppl. 1964; 65(65):1-109.

3. Lawton JO, Baker MR, Dickson RA. Femoral neck fractures. Two populations. Lancet. 1983; 2:70-2. 
4. Tinetti ME, Doucette JT, Claus EB. The contribution of predisposing and situational risk factors to serious fall injuries. J Am Geriatr Soc. 1995; 43:1207-1213.

5. Murray RC, Frew JFM, Inverness, Scotland. Trochanteric fractures of the femur-a plea for conservative treatment. J Bone Joint Surg. 1949; 31:205-219.

6. Bannister G, Gibson A, Ackroyd C, Newman J. The fixation and prognosis of trochanteric fractures. Clin Orthop Relat Res, 1990; 254:242-6.

7. Obrant KJ, Bengner U, Johnell O. Increasing age adjusted risk of fragility fractures: a sign of increasing osteoporosis in successive generations? Calcif Tissue Int. 1989; 44:157-67.

8. Adams CI, Robinson CM, Court-Brown CM et al. Prospective randomized controlled trial of an intramedullary nail versus dynamic screw and plate for intertrochanteric fracture of the femur $[\mathrm{J}]$. J Orthop Trauma. 2001; 15(6):394-400.

9. McLoughlin SW, Wheeler DL, Rider J, Bolhofner B. Biomechanical evaluation of the dynamic hip screw with two and four hole side plates. J Orthop Trauma. 2000; 14(5):318-23.

10. Dean GL, David SG, Jason HN. Osteoporotic pertrochanteric hip fractures: management and current controversies. J Bone Joint Surg [Am]. 2004; 86:398-409.

11. Wagner R, Weckbach A, Sellmair U, Blattert T. Extraarticular proximal femur fracture in the elderly - dynamic hip screw or intramedullary hip screw for management? Langenbecks Arch Chir Suppl Kongressbd. 1996; 113:963-6.

12. Sommer C, Gautier E, Muller M, et al. First clinical results of the locking compression plate (LCP). Injury 2003; 34: S-B43-54.

13. Sperner G, Wanitschek P, Benedettok K, Glotzer W. Technical errors and early complications of osteosynthesis of pertrochantric fractures using dynamic hip screw. Unfallchirurg. 1989; 92:571-6.

14. Egol KA, Kubiak EN, Fulkerson E, Kummer FJ, Koval KJ. Biomechanics of locked plates and screws. J Orthop Trauma. 2004; 18(8):488-93.

15. Korner J, Diederichs G, Arzdorf M, Lill H, Josten C, Schneider E et al. A biomechanical evaluation of methods of distal humerus fracture fixation using locking compression plates versus conventional reconstruction plates. J Orthop Trauma. 2004; 18(5):286-93.

16. Mueller KL, Karunakar MA, Frankenburg EP et al. Bicondylar tibial plateau fractures: a biomechanical study. Clin Orthop Relat Res. 2003; 412:189.

17. Jewell DP, Gheduzzi S, Mitchell MS. Locking plates increase the strength of dynamic hip screws [J]. Injury 2008; 39(2):209-12.

18. Wagner M. General principles for the clinical use of the LCP. Injury. 2003; 34(2):31-42.

19. Perren S, Cordey J, Rahn B. Early temporary porosis of bone induced by internal fixation implants. A reaction to necrosis, not to stress protection? Clin Orthop Relat Res. 1988; 232:139-51.

20. Ecker Malcolm L. The treatment of trochanteric fractures using compression screw. J. Bone Jt. Surg. Am. 1975; 57A:1-22.

21. Rao JP, Banzon MT, Weiss AB, Rayhack J. Treatment of unstable intertrochanteric fractures with anatomic reduction and compression hip screw fixation. Clinical orthopaedics and related research. 1983; 175:65-71.

22. Bridle SH, Patel AD, Bircher M, Calvert PT. Fixation of intertrochanteric fractures of the femur. J. Bone Joint. Surg. 1991; 73-B:330-334.

23. Baumgaertner MR, Curtin SL, Lindskog DM. Intramedullary versus extramedullary fixation for the treatment of intertrochanteric hip fractures. Clin Orthop 1998; 348:87-94. 